\title{
LA HIPERGLUCEMIA DE ESTRÉS, DE CLAUDE BERNARD AL NICE-SUGAR, UN OBJETIVO EN MOVIMIENTO
}

\author{
THE STRESS HYPERGLYCEMIA, FROM CLAUDE BERNARD \\ TO NICE-SUGAR, A MOVING TARGET
}

Sabemos que el control glucémico se deteriora en forma significativa durante la internación de un paciente con diabetes mellitus (DM). Contribuyen a la hiperglucemia factores como el estrés, tanto psíquico como físico, tratamientos concomitantes, disminución de la actividad física, nutrición enteral o parenteral, medicamentos como los corticoides y el temor a las hipoglucemias por parte del médico, mientras que en la hipoglucemia intervienen factores como la disminución de la ingesta calórica, enfermedades gastrointestinales, errores en la medicación y alteraciones cognitivas ${ }^{1}$. Aunque también puede existir aumento de la glucemia en pacientes sin DM previa; esta hiperglucemia causada por enfermedades se denomina hiperglucemia de estrés.

Las enfermedades producen aumento de las hormonas de contrarregulación, como cortisol y adrenalina, lo que conduce a un aumento de la producción hepática de glucosa, disminución de su captación a nivel muscular e incremento de la lipólisis; todo esto provoca un aumento de la glucemia, ácidos grasos libres, lactato y cetonas con el consiguiente deterioro de la enfermedad lo que genera un bucle que determina un incremento de la estadía hospitalaria, discapacidad y posibilidad de muerte.

La hiperglucemia en internación se define como aquella mayor a $140 \mathrm{mg} \%$, y es muy frecuente tanto en sala general como en áreas críticas, incluso está presente hasta en un $80 \%$ de los pacientes en algunos estudios en críticamente enfermos ${ }^{2}$.

Se conoce la asociación de la hiperglucemia con complicaciones hospitalarias, incluyendo la mortalidad, tanto en personas con en aquellas sin DM previa, siendo más frecuente en estas últimas ${ }^{3-4}$. El primero en describir la hiperglucemia de estrés fue el fisiólogo Claude Bernard en el año 1877, aunque esto no significó ningún cambio hasta que a fines del siglo XX y principios del XXI comenzaron a desarrollarse múltiples estudios sobre el tema.

Uno de los primeros fue el estudio Diabetes Mellitus, Insulin Glucose in Acute Myocardial Infarction (DIGAMI) que en el año 1997 evaluó la disminución en la mortalidad de pacientes tratados con insulinoterapia intensificada luego de un infarto agudo de miocardio, y demostró una reducción del RR del 28\% a los cinco años a favor del tratamiento intensivo $(p=0,011)^{5}$. En 1999 se publicó el estudio "Portland" que señaló una disminución estadísticamente significativa de las infecciones profundas a nivel de las esternotomías después de una cirugía cardíaca con el tratamiento intensivo de las hiperglucemias RR 0,34 (IC 0,14$0,74 p=0,01)^{6}$. En ambos estudios el objetivo fue mantener las glucemias por debajo de $200 \mathrm{mg} \%$.

En el año 2001 se publicó el estudio "Leuven" en pacientes críticamente enfermos de una UTI quirúrgica que tenían como objetivo glucemias entre 80 y $110 \mathrm{mg} / \mathrm{dl}$ en el grupo de tratamiento intensivo, y presentaron una disminución de mortalidad en UTI e intrahospitalaria del 42 y $34 \%$ respectivamente $(p=0,04$ y $p=0,01)$. Este estudio también expresó diferencias estadísticamente significativas a favor del tratamiento intensivo en bacteriemia, uso prolongado de ATB, empleo de ventilación mecánica, diálisis y estadía prolongada en UTI, y fue por muchos años el que marcó el objetivo glucémico en los pacientes críticos?.

En 2005 y 2006 se intentaron reproducir estos estudios con diferentes poblaciones, realizando el DIGAMI II y Leuven II, sin conseguir resultados similares ${ }^{8-9}$.

Después de ocho años de la aparición del estudio Leuven, se publicó el estudio Normoglycemia in Intensive Care Evaluation-Survival Using Glucose Algorithm Regulation (NICE-SUGAR) que propuso un objetivo glucémico entre 140 y 180 mg/dl, y de- 
mostró una disminución absoluta de la mortalidad del $2,6 \%$ a favor del tratamiento convencional comparado con el tratamiento intensivo (TI) $(95 \% \mathrm{IC}, 0,4-$ 4,8), Odds Ratio para muerte con $\mathrm{Tl} 1,14(95 \% \mathrm{Cl}$, 1,02-1,28; $p=0,02$ ), cuyos objetivos glucémicos se establecieron entre 81 y $108 \mathrm{mg} / \mathrm{dl}$, planteando que este aumento en la mortalidad se daba por un incremento significativo de las hipoglucemias moderadas y severas en el grupo de $\mathrm{TI}^{10}$.

Todos estos estudios y otros, como el GlucoCABG trial publicado en 2015, hicieron que la American Association of Clinical Endocrinologists y la American Diabetes Association (AACE/ADA) en el Consenso de 2016 recomendaran un objetivo glucémico entre 140 y 180 mg/dl en los pacientes críticamente enfermos con un grado de evidencia " $A$ ", y entre 110 y 140 mg/dl en pacientes seleccionados en áreas críticas sin riesgo significativo de hipoglucemia (IAM, ACV y cirugía cardiaca) $)^{11-12}$.

En esta edición nos encontramos con un trabajo del Dr. Guillermo Alzueta y colaboradores, realizado en el Capítulo Atlántico de nuestra Sociedad entre los años 2000 y 2001, que muestra datos muy similares a los que tiempo después fueran publicados en diferentes partes del mundo, teniendo como aporte novedoso la asociación de la mortalidad de los pacientes hiperglucémicos con insuficiencia cardíaca comparados con aquellos no hiperglucémicos, además de brindarnos datos estadísticos respecto del tema en un sector de nuestra población del cual contamos con escasa información.

\section{BIBLIOGRAFÍA}

1. Metchick LN, et al. Inpatient management of diabetes mellitus. Am J Med 2002; 113:317-323.

2. Kosiborod M, Inzucchi S, Clark B, et al. National patterns of glucose control among patients hospitalized with acute myocardial infarction (abstract 1018-183). J Am Coll Cardiol 2007; 49(9 suppl 1):283A-284A.

3. Frisch A, et al. Diabetes 58 (suppl 1) A27, 2009.

4. Kosiborod M, et al. Glucometrics in patients hospitalized with acute myocardial infarction: defining the optimal outcomes-based measure of risk. Circulation 2008; 117:1018-27.

5. Malmberg K, et al. Prospective randomised study of intensive insulin treatment on long term survival after acute myocardial infarction in patients with diabetes mellitus. DIGAMI (Diabetes Mellitus, Insulin Glucose Infusion in Acute Myocardial Infarction) Study Group. BMJ 1997 May; 24, 314(7093):1512-5.

6. Furnary AP, et al. Continuous intravenous insulin infusion reduces the incidence of deep sternal wound infection in diabetic patients after cardiac surgical procedures. Ann Thorac Surg 1999; 67:352-362.

7. Van den Berghe G, Wouters P, Weekers F, et al. Intensive insulin therapy in critically ill patients. $N$ Engl J Med 2001; 345:1359-1367.

8. Malmberg K, et al. Intense metabolic control by means of insulin in patients with diabetes mellitus and acute myocardial infarction (DIGAMI 2): effects on mortality and morbidity. Eur Heart J 2005 Apr; 26(7):650-61.

9. Van den Berghe $\mathrm{G}$, et al. Intensive insulin therapy in the medical ICU. N Engl J Med 2006 Feb 2; 354(5):449-61.

10. Finfer $S$, et al. Intensive vs conventional glucose control in critically ill patients. N Engl J Med 2009 Mar 26; 360(13):1283-97.

11. Umpierrez GE, et al. Randomized controlled trial of intensive vs conservative glucose control in patients undergoing coronary artery bypass graft surgery: GLUCOCABG Trial. Diabetes Care 2015; 38:1665-1672.

12. 2016 ADA Standards. Diabetes Care 2016; 39 (Suppl 1):S99-S104.

Dr. Jorge Fabián Tedesco

Especialista Universitario en Diabetes (UNC) Especialista en Clínica Médica

Médico de Planta del Servicio de Endocrinología,

Diabetes y Nutrición del Hospital

San Martín, Paraná, Entre Ríos 